\title{
PHYLLANTHUS SP A LOCAL PLANT WITH MULTIPLE MEDICINAL PROPERTIES
}

\author{
Tang YQ, Lee SH, Sekaran SD \\ Department of Medical Microbiology, Faculty of Medicine, University of Malaya, Kuala Lumpur
}

\section{Correspondence:}

Shamala Devi Sekaran

Department of Medical Microbiology, Faculty of Medicine,

University of Malaya, 50603 Kuala Lumpur, Malaysia

E-mail: shamalamy@yahoo.com

Telephone: +603-79675759

Fax: $+603-79676672$

\begin{abstract}
The plants of the genus Phyllanthus (Euphorbiaceae) are distributed in most tropical and subtropical regions of world. This plant has been long used as a traditional medicine to treat problems such as stomach, intestinal infections, kidney and urinary bladder disturbances, diabetes, and hepatitis B. There has been considerable interest in these plants in recent years. This review discusses the antiviral and anticancer aspects of Phyllanthus species. Scientific studies have demonstrated that extracts and purified isolated compounds (flavonoids, lignans, phenols, and terpenes) obtained from these plants possess antiviral effects against herpes simplex (HSV) and dengue virus infections (DENV). These observations are associated with the disruption of essential proteins needed during viral cycle, thus halting the viral replication. In addition, the Phyllanthus species have also been shown to exert inhibitory effects against selected cancers types. In these studies anti-proliferative, anti-metastatic, anti-angiogenic effects and induced apoptosis of human cancers cell lines were observed. These may be explained by the disruption of multiple survival pathways and differential protein expression. CONLCUSION:As a conclusion, tThe Phyllanthus plant possesses multiple medicinal properties, including antiviral and anticancer activities which may potentially be used as a medicinal source for many disease locally.
\end{abstract}

Keywords: anticancer, antiviral, broad spectrum antimicrobial, dengue, Phyllanthus

\section{Phyllanthus}

Besides being sources of food supply, plants and herbs also play important roles in the treatment of illnesses indigenously (1-3). One of the best examples is the discovery and development of Artemisinin (antimalaria drug) from Artemisia annua $L$ (4). Since our ancestral times, they have discovered and primarily used a vast range of natural compounds to improve healing or treat diseases and injuries. It is only during the past decades where the importance of natural products has emerged second to the designing of synthetic compounds. However, scientists have found a new role for natural products as a basis for drug development and hence a renewed attention to natural products has emerged (5).

Phyllanthus is a small annual plant that is widely distributed throughout the tropical and subtropical regions of world (6). It is a very large genus containing approximately 550-750 species including $P$. accuminatus, $P$. amarus, P. pulcher, P. niruroides, P. anisolobus, P. orbiculatus, $P$. emblica, $P$. oxyphyllus, $P$. flexuosus, $P$. raticulatus, $P$. fraternes, $P$. simplex, $P$. mullernus, $P$. urinaria, $P$. mytrifolis,
P. virgatus, P. niruri and P. watsonii (7-11). In Ayurvedic medicine, Phyllanthus has a long tradition of use to treat jaundice, gonorrhea, frequent menstruation, dysentery and diabetes, as well as skin ulcers, sores, swelling, and itchiness (8). In Traditional Chinese Medicinal (TCM), Phyllanthus has been used to treat gallstones and kidney stones. In addition, this plant has been thought to stimulate the immune system (9).

The bioactive components present in various plants and foods have a prominent role for diseases prevention. Phyllanthus extracts have been reported to have several beneficial pharmacological effects, which include antiviral activity against hepatitis $B$ and related hepatitis viruses. Others have also reported that the plant provides antibacterial, antihepatotoxic, and antidiabetic activities $(10-11)$. This could be associated with the present of different bioactive compounds such as geraniin, gallic acid, rutin and quercetin (10-15).

It has been demonstrated that these plants are potentially good sources for the production of antiviral agents as compared to synthetic analogues. More interestingly is 
that by using plants as the source of antiviral agents, supply would not be a problem since the majority of these plants are usually found abundantly in developing and third world countries, where coincidentally, the incidences of infectious diseases are more widespread. Many of these diseases are still waiting for a miraculous vaccine or drug. Among these include herpes simplex (HSV) and dengue (DENV) virus infections. Besides that, plant has also been proposed by various research groups to demonstrate anticancer activities on different cancers $(8,9)$. In this particular review, the antiviral and anticancer aspects of Phyllanthus species carried out in our laboratory are highlighted.

\section{Antiviral activities of Phyllanthus}

\subsection{Anti-Herpesviridae (HSV) Properties}

\subsubsection{Introduction on HSV}

Herpesviridae family consists of at least eight species of single large double stranded DNA enveloped viruses that are pathogenic to humans (16-20). Among these species, HSV-1 and HSV-2 are more intriguing due to the enormous clinical symptoms that they may manifest, ranging from gingivostomatitis to keratoconjunctivitis, genital disease, encephalitis. In addition, these viruses are likely to infect newborn and immunocompromised patients, which is more alarming (20). The main concern however, are their ability to remain latent and produce lifelong infections, get reactivated whenever triggered by a particular stimuli; which can include sunlight, stress or due to the weakened immunity, resulting in secondary infections (21).

\subsubsection{Treatments of Herpes Simplex Virus}

The primary treatment for HSV infections involves mainly nucleoside analogs such as acyclovir, valaciclovir, famciclovir and cidofovir. The only drawback of these limited anti-HSV drugs is the development of viral resistance against these treatments and drug toxicity (19-21). Drug-resistant viral strains were increasingly isolated from patients, in particular immunocompromised individuals (20). Although more HSV inhibitors (both nucleoside and nonnucleoside analogues) were established, very few of them were approved for therapeutic use due to their relatively high toxicity (20). Thus, it is imperative to search for new antiherpetic agents that are less toxic and have lower probability of resistance development for the prevention or treatment of herpes virus infections.

\subsubsection{Role of Phyllanthus species in HSV infection}

\subsubsection{Phyllanthus orbicularis}

In 2003, Fernandez Romero and his research group prepared two butanol- and acetic acid-soluble fractions from the leaves and stems of Phyllanthus orbicularis and tested these fractions against acyclovir-sensitive and -resistant HSV-1 strains in both human foreskin fibroblast (HFF) and green ape kidney (Vero) cell lines. Both the fractions exhibited antiviral selectivity indexes (SI) ranging from 10.3 to 22.8 , while their extracellular virucidal activities reached SI values ranging from 371 to 1040 (22). In another study, a fraction that contains flavan-3-ol gallates, procyanidin B1 and B2, procyanidin dimer gallates, procyanidin trimers and procyanidin trimer gallates exerted strong anti-HSV-2 activity. As compared to this fraction though, its crude methanol extract showed higher antiviral selectivity index, suggesting the loss of some active components contributing to the antiviral activity during separation (23). The third fractionation studies revealed inhibition of acetic ether, methanol and aqueous extracts from Phyllanthus emblica on HSV-1 at $\mathrm{IC}_{50}$ values $25.28,66.17$ and $100.94 \mu \mathrm{g} / \mathrm{ml}$, respectively. Whereas on HSV-2, the IC $\mathrm{C}_{50}$ value of acetic ether, methanol and aqueous extracts from Phyllanthus emblica are 31.7, 180.3 and $112.1 \mu \mathrm{g} / \mathrm{ml}$, respectively (24).

\subsubsection{Phyllanthus urinaria}

Subsequent studies involved isolation of pure compounds from acetone extract (leaves and branches) of Phyllanthus urinaria and their results showed that hippomanin A blocked only HSV-2 infection but corilagin inhibited neither HSV-1 nor HSV-2 replication although both these compounds displayed similar structures (25). Another polyphenolic compound, 1,2,4,6-tetra-O-galloyl- $\beta$-d-glucose (1246TGG) isolated from Phyllanthus emblica was found to inhibit both HSV-1 and HSV-2 infections at varying magnitudes of activity in vitro. This compound has been shown to inactivate HSV-1 particles directly, thus this leads to the inhibition of viral attachment and penetration during early infection. Other anti-HSV mechanisms of 1246TGG include an antiviral effect mainly within 3 hours post-infection as well as intracellular growth suppression of HSV-1 up to 12 hours post-infection. This is mainly due to the inhibition of HSV-1 E and L gene expressions and viral DNA replication without interrupting the RNA synthesis of IE gene, thus this leads to the reduction of viral protein synthesis. Taken together, 1246TGG might exert anti-HSV activity both by inactivating extracellular viral particles and by inhibiting viral biosynthesis in host cells (26). A similar structure purified from the acetone extract of Phyllanthus urinaria, 1,3,4,6-tetra-O-galloyl- $\beta$-d-glucose (1346TOGDG) effectively reduced HSV-1 infection with $\mathrm{IC}_{50}$ values of $19.2 \pm 4.0 \mu \mathrm{M}$. Geraniin, a more well established pure compound isolated from the same extract actively suppressed HSV-2 infection with $\mathrm{IC}_{50}$ values of $18.4 \pm 2.0 \mu \mathrm{M}(27)$.

Activities of three Phyllanthus urinaria (whole plant) extracts, acetone, ethanol and methanol, inhibited HSV-2 infections at $I C_{50}$ values of $4.3 \pm 0.5,5.0 \pm 0.4$ and $4.0 \pm 0.9$ $\mathrm{mcg} / \mathrm{ml}$, respectively. All three extracts showed no cytotoxic effect against Vero cells at concentrations of 10.0 $\mathrm{mcg} / \mathrm{ml}$ and below. However, the time-of-addition study showed that these three extracts were only effective during simultaneous treatment, suggesting that they affect the initial stage of HSV-2 infection (20). Similar phenomenon was observed in Tan et al's (28) study whereby two mode 
of treatments (simultaneous- and post-treatments) of four whole Phyllanthus plant (P.amarus, P.niruri, P.urinaria and P.watsonii) aqueous extracts effectively inhibited both HSV-1 and HSV-2 via inhibition of viral entry, binding and replication. Pure isolation of excoecarianin from the acetone extract of whole Phyllanthus urinaria plant protected Vero cells from HSV-2 infection with $\mathrm{IC}_{50}$ values $1.4 \pm 0.1 \mu \mathrm{M}$ without affecting the viability or the morphology of Vero cells. This inhibitory effect was most prominent when excoecarianin was added concurrently with the virus as compared to pre-treatment and post-viral entry treatment that did not yield any antiviral effects. This revealed that excoecarianin primarily inactivate HSV-2 virus particles to prevent viral infection. It was also able to act synergistically with acyclovir to exhibit antiviral effect against HSV-2 infection in Vero cells (21).

\subsubsection{Phyllanthus emblica}

The effects of pentagalloylglucose (PGG), a hydrolyzable polyphenol isolated from the branches and leaves of Phyllanthus emblica were tested on HSV-1-infected MRC-5 cells with or without PGG-treatment, as well as between non-infected MRC-5 cells with or without PGG-treatment by two-dimensional gel electrophoresis coupled to mass spectrometry analysis. Significantly, PGG downregulated cofilin1, a key regulator of actin cytoskeleton dynamics which is important for HSV-1-induced actin-skeleton rearrangements for infectivity, leading to a reduced HSV1 DNA, mRNA, protein synthesis and virus yields (29). Other antiviral mechanisms of the active Phyllanthus orbicularis (aerial parts) extracts and fractions involved inhibition of several HSV-2 early replication events and DNA synthesis (30).

\subsubsection{Summary}

Of importance it is observed that both whole extract and pure compounds derived from Phyllanthus plants exerted very minimal toxicity without changing cellular morphologies or affecting cells viability $(8-9,17,20-22$, 25-28). Various pure compounds were successfully isolated from the Phyllanthus extracts, each of these compounds demonstrating specific inhibitory activities towards the different herpes simplex viruses, unlike the whole extract that possessed a broader spectrum of antiviral activity against both HSV-1 and HSV-2. Nonetheless, most of these research groups reported that whole Phyllanthus plant extracts inhibits the herpes simplex viruses most effectively at the time of infection (simultaneous treatment). They postulated the blocking of viral entry or replication during early HSV infection as the antiviral mechanisms. In actual case scenario however, majority of HSV infections were only diagnosed in patients present with clinical symptoms, signifying a post-infection stage rather than simultaneous infection. In such circumstance, Phyllanthus might not be a valuable anti-HSV agent and hence, further assessment of Phyllanthus in other pharmacological effects is essential before it could attract public interest as a plant possesses multiple medicinal properties.

\subsection{Anti-Dengue Properties}

\subsubsection{Introduction on Dengue}

Dengue is a globally important mosquito-borne arboviral infection that has spread to many tropical and subtropical areas, endangering an estimated 2.5 billion people and hence causing an increasing public health concern in endemic countries. There is approximately 50 to 100 million infections reported each year, of which 500,000 cases were severe and potentially life-threatening $(31,32)$. The causative agent for dengue disease is an icosahedral, enveloped virus that contains a single-stranded positive sense genome (31). Due to the serological and genetic relatedness, dengue virus (DENV) can be grouped into four serotypes: DENV-1 to DENV-4. It appears that all of these variants can co-circulate in endemic areas. Infection by a particular serotype confers lifelong protection against similar serotype, however protection against secondary infection by another heterologous serotype is only temporary $(32,33)$. Human population of all ages are susceptible to dengue virus infection, leading to diverse illness symptoms, often involving an abrupt onset of fever, myalgia, headache, severe retro-orbital pain, and petechiae. In a more severe scenario, the individual might present with capillary leakage, hemorrhage, and circulatory shock $(31,32)$. Severe disease is mainly observed in secondary, heterologous DENV infections. The mechanism by which this occurs can be explained using the antibodydependent enhancement (ADE) of infection theory, which clearly describes the primary mechanism of dengue immunopathogenesis (33). Recognition of these clinical symptoms is crucial for successful patient management, mainly involving careful fluid infusion (31).

As specific dengue treatments are not yet available, dengue vaccine development is considered a major advance in the disease control. The only drawback for this vaccine development is the potentially detrimental role of immune enhancement (33). Therefore, the need for new antiviral agents is of paramount in light of the need to prevent the manifestation of severe dengue. Further, this will also ensure that outbreaks can be prevented and, in future to complement possible vaccination programs. The therapeutic properties against dengue infection is currently still in its early stages. However, understanding of the molecular mechanisms behind the dengue virus life cycle and elucidation of the specific functions for each dengue viral proteins has allowed the design of novel antiviral targets. Some of these potential therapeutic intervention targets include viral entry, viral RNA polymerase/ methyltransferase, nucleotide synthesis, viral serine protease, $\alpha$-glucosidases and kinases (34). Nonetheless, plants that are rich in bioactive components remain the basis for these novel antiviral compounds. 


\subsubsection{Antiviral activity of Phyllanthus}

Klawikkan and his research group first investigated the effects of various medicinal plant extracts from Thailand against DENV2 in Vero cell. Amongst these plant extracts, dichloromethane and ethanol extracts of Phyllanthus urinaria did not exhibit any DENV inhibitory activity when tested at $12.5 \mu \mathrm{g} / \mathrm{ml}$ (35). Two years later, cocktail (aqueous and methanolic) extracts were prepared from whole four Phyllanthus plants ( $P$. amarus, P. niruri, $P$. urinaria, and $P$. watsonii). The maximum non-toxic dose on Vero cells for both aqueous and methanolic extracts were determined to be $250 \mu \mathrm{g} / \mathrm{ml}$ and $15.63 \mu \mathrm{g} / \mathrm{ml}$ respectively. At these concentrations, Phyllanthus showed strongest inhibitory activity against DENV2 with more than $90 \%$ of virus reduction in simultaneous treatment through regulation of various proteins that play important roles in cell-virus attachment, viral entry, viral polyprotein production, viral RNA replication as well as viral assembly and maturation $(17,37)$. Inhibition of the viral entry into the host cell via binding of viral $\mathrm{E}$ protein to heparin sulfate during early infection is normally the first point of intervention (34).

\subsubsection{Larvicidal activity of Phyllanthus}

Management of dengue disease vectors provides a better means of controlling disease outbreak. Transmission of all four dengue virus serotypes to humans occurs via infected Aedes spp. mosquito bite, mainly Aedes aegypti, an anthropophilic mosquito. Outbreak transmission could also be caused by other members including A. albopictus, A. polynesiensis, $A$. mediovittatus and members of the $A$. scutellaris. Hence, knowledge of mosquito vectors and the interactions between virus and vector is essential to establish methods to interrupt transmission, for instance, by targeting elimination or suppression of target mosquito populations in endemic areas (34). It is envisaged from studies conducted using Phyllanthus that it has a putative role in reducing viral load in mosquitoes. In 2012, Sarita evaluated the larvicidal activity of 15 Indian plant species and the preliminary screening showed that only 10 plants possessed larvicidal potential as they could result in $100 \%$ mortality at $1000 \mathrm{ppm}$. Among these plants, they showed that the hexane extract from Phyllanthus emblica fruit was the least effective with an LC $_{50}$ value of $298.93 p p m$. However, Jeyasankar and his group performed a similar experiment by examining the mosquitocidal potential of Phyllanthus emblica against $A$. aegypti and his results obtained were more promising. The hexane extract from the leaves of Phyllanthus emblica was tested against the fourth instar larvae of $A$. aegypti, where $86.0 \%$ of the larval mortality was observed at $250 \mathrm{ppm}$ concentration with the $\mathrm{LC}_{50}$ of 111.34ppm ( $\left.L C L=93.07-\mathrm{UCL}=133.20\right)$. Meanwhile, the ethyl acetate extract of Phyllanthus emblica exhibited the maximum larvicidal activity (99.6\% larval mortality) with $\mathrm{LC}_{50}$ value of 78.89ppm (37).

In summary, Phyllanthus has the potential to be a candidate in the development of anti-dengue agents. It may work either by causing toxicity to the mosquito larvae as a part of vector control management, or by inhibiting the infection of dengue virus in the host cells. Different parts of the same Phyllanthus species may also give rise to the different magnitude of anti-dengue effects, probably attributed to the varying composition of bioactive components present. Nevertheless, the in vitro antiviral activities of Phyllanthus studied so far focused only on a particular dengue virus serotype (DENV-2) and more detailed investigations on other DENV serotypes is crucial since these serotypes may present different infectivity and virulence. Besides that, in vivo (toxicity and efficacy) investigations and pre-clinical studies need to be conducted in order for the plant to be accepted as a prospective anti-dengue agent.

\section{Anticancer activities of Phyllanthus}

\subsection{Cancer}

Cancer refers to a group of diseases that arise from a single (mutated) cell when it starts to grow uncontrollably to form a tumor (group of undifferentiated cells). Different stages of the tumor exhibit different responses to treatment thus making treatment very difficult. Most of the chemotherapeutic drugs including doxorubicin and cisplatin, mainly affect the fast-dividing cells of the body causing undesirable side effects such as pain, nausea, vomiting, alopecia, and anaemia (38).

Evasiveness of apoptosis is a hallmark of cancer and is critical for cancer development and survival of tumor cell (3). This has led the cancer cells to possess uncontrolled proliferation and form a large mass of abnormal (mutated) cancer cells: tumor. During transformation of malignant tumor, tumor will triggers angiogenesis: a process whereby new blood vessels are formed from pre-existing blood vessels, to provide the route for tumor cells to exit from the primary tumor and enter the circulation and spread to other parts of the human body. Therefore, angiogenesis and metastases are intrinsically connected. This ability of malignant tumors to metastasize is responsible for the poor prognosis and the apparent high mortality rate in cancer patients (3). Therefore, metastasis and angiogenesis remain a major clinical challenge in cancer treatment.

Development of chemoresistance to the anticancer drugs by cancer cells is another major obstacle in cancer treatments and has resulted in high mortality rate among cancer patients. Currently, there is no effective treatment for cancer, so intense research is required to obtain new anticancer agents for this cancer. Thus, scientists have begun to focus on natural product as an alternative to cancer treatment following successful development of natural-based anticancer agents such as zapotin, apigenin and resveratrol $(38,39)$.

\subsection{Anti-proliferative effect}

The anti-proliferative effects of four whole Phyllanthus plants (P.amarus, P.niruri, P.urinaria and P.watsonii) have been identified in different cancer cell lines including breast, lung, melanoma, liver, lung, leukemia and prostate 
$(8,9,40-44)$. The selective anti-proliferative effect of Phyllanthus was observed when whole extract of four Phyllanthus species (P.amarus, P.niruri, P.urinaria and P.watsonii) inhibited growth of four different cancer cell lines; A549, MeWo, MCF-7 and PC-3, without cytotoxic effects on their respective normal cells $[8,9,40]$. Most of the polyphenol compounds present in Phyllanthus plant have been reported to possess anti-proliferative effects on cancer cells including gallic acid (44).

The anti-proliferative effect of anticancer agents has also been associated with cancer cell cycle arrest (G0/G1, S or $\mathrm{G} 2 / \mathrm{M}$ ) that finally leads them to apoptotic cell death (45-46). Phyllanthus plants have been reported to induce cell cycle arrest in cancer cells, which leads to inhibition of cancer cell proliferation and eventually induces apoptosis.
The whole plant extract of four Phyllanthus species (P.amarus, P.niruri, P.urinaria and P.watsonii) have been reported to induce $\mathrm{G} 1$ - and S-phase arrest in PC-3 and MeWo cells, respectively (40). For instance, the G1-phase arrest in Phyllanthus treated PC-3 cells was believed to be due to the disruption on several cellular pathways including MAPKs, Wnt, NFkB and Myc/Max (Figure 1) (8). Downregulation of these pathways could lead to the activation of 27 proteins, which in turn inhibit the activation of cyclin E/CDK2 and/or cyclin D/CDK4 complexes, thus halting cell cycle progression at $\mathrm{G} 1$ phase (47). In addition, degradation of c-myc and $\beta$-catenin proteins by GSK3 $\beta$ could reduce the production of cyclin $D$, which is an important initiator of cell cycle. Several studies have shown the involvement of Wnt and Myc/Max pathways in the regulation of cyclin D to induce cell growth arrest (Figure 1) (8, 47-49).

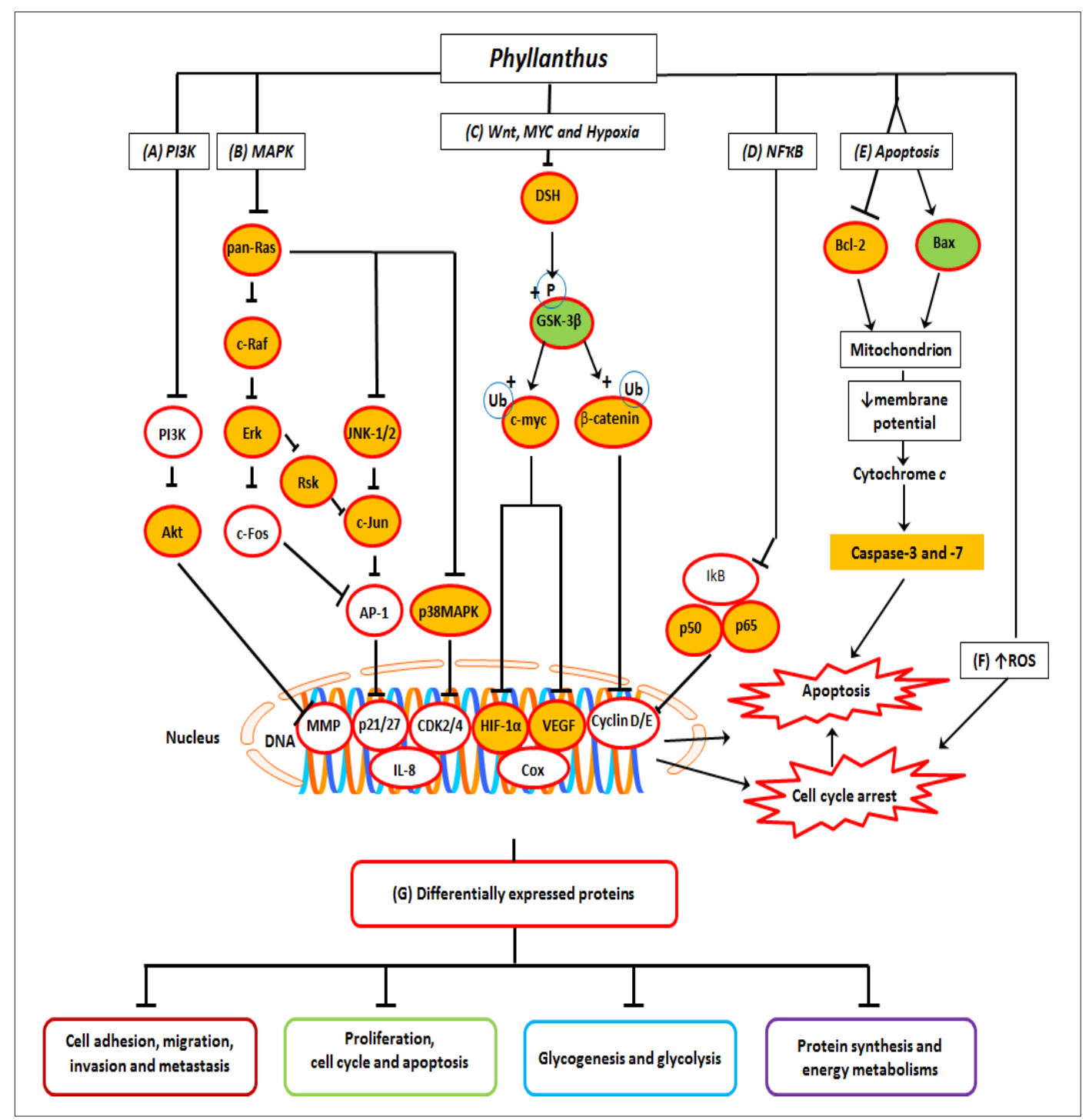

Figure 1: Schematic diagram illustrating that Phyllanthus regulates multiple survival signalling pathways and protein activities in cancer cells. The inhibition of (A) PI3K/Akt (Akt protein), (B) MAPKs (pan-Ras, c-Raf, RSK, Elk1, c-Jun, JNK1/2, and p38 MAPK proteins), (C) Wnt (DSH, Gsk3, and -catenin proteins), Myc/Max (c-myc protein), hypoxia (HIF-1 $\alpha$ and VEGF proteins) and (D) NFKB (p50 and p52 proteins) pathways by Phyllanthus plant extracts in cancer cells. (E) Apoptosis induction via up-regulation of Bax protein and down-regulation of $B c l-2$ to induce caspase-3/7 activation in treated cancer cells. (F) Induction of reactive oxidative stress by Phyllanthus leads to cell cycle arrest and apoptosis. (G) A number of proteins involved in proliferation, cell cycle, apoptosis, metastasis, glycogenesis and glycolysis, protein synthesis as well as energy metabolism were found altered in cancer cells upon Phyllanthus treatment. 


\subsection{Apoptosis inducer}

Whole extract of Phyllanthus plants (P.amarus, P.emblica, P.niruri, P.urinaria and P.watsonii) have been reported to induce apoptosis in various types of cancer cells including melanoma, breast, lung, colorectal, cervical, liver and prostate $(8,9,40,44,50-55)$. Apoptosis induction by these Phyllanthus is always associated with the activation of effector caspases such as caspase-3 and -7. These activated effector caspases will in turn activate other degradative enzymes such as DNases to cleave the DNA into fragments in an apoptotic cell. Besides involvement of caspases activation, Phyllanthus plant has been reported to utilize other mechanisms to induce apoptosis in cancer cells. For instance, the methanolic extract of hairy root of P.amarus increased the levels of intracellular reactive oxygen species (ROS) and decreased mitochondrial membrane potential (MMP) to induce apoptosis in MCF-7 (50). In addition, whole plant extract of P.amarus, P.niruri, P.urinaria and P.watsonii have also been reported to increase the expression of pro-apoptotic protein, Bax, and reduce expression of anti-apoptotic protein, $\mathrm{Bcl}-2$, which in turn allow apoptosis to occur in cancer cells (Figure 1) $(8,51$ 52). In addition, whole plant extract of three Phyllanthus species ( $P$. urinaria, $P$. amarus and $P$. debilis) also induced TNF- $\alpha$ production and inhibited expression of other anti-apoptotic genes including IL-8 and COX-2 in human hepatocarcinoma cells (43).

\subsection{Anti-angiogenic and anti-metastatic effects}

Endothelial cells are primary cells that form the lining of blood vessels. Although Phyllanthus showed no cytotoxic effect on endothelial cells, it decreased the migration and invasive ability of endothelial cells, thus inhibiting the formation of new blood vessels (41). The anti-angiogenic effect of water extract of whole Phyllanthus plant was observed when it inhibited microcapillary tube-like formation of endothelial cells cultured on extracellular matrix (ECM), which mimics the in vivo lining of blood vessels and in addition, it decreased the vessel density in both in vivo and ex vivo studies (53).

Phyllanthus plants have also shown the potential to reduce the metastazing ability of several cancer cell lines $(8,9$, 54-55). For example, gallic acid found in Phyllanthus plant has disrupted cancer cell-cell interaction in a mechanical scratch-wound cellular monolayer healing assay (8, 9, 54). In addition, whole Phyllanthus urinaria extract also inhibited the invasion ability of cancer cells in a dosedependent manner through the ECM gel as matrix barrier, which mimiced the in vivo basement membrane of blood vessel $(41,55,56)$. This anti-metastatic effect of whole Phyllanthus plant extracts (P.amarus, P.emblica, P.niruri, P.urinaria and P.watsonii) was further observed when Phyllanthus showed inhibitory effects on different matrix metalloproteinases (MMPs) in various types of cancer cells $(8, \ldots, 53,56-57)$. Four whole plant extracts of Phyllanthus (P.amarus, P.niruri, P.urinaria and P.watsonii) were noted to inhibit the glycolytic pathway and energy production in PC-3 cells by down-regulating HIF- $1 \alpha$ protein which leads to reduction of pro-angiogenic VEGF and thus inhibit tumor angiogenesis and thereby decrease cancer progression (Figure 1) (8).

\subsection{Anticancer overview}

In summary, cumulative results from experimental and predictive studies suggest that whole plant extract of Phyllanthus can interfere with (1) multiple survival signalling pathways (PI3K/Akt, MAPKs, Wnt, Myc/Max, hypoxia and NFkB), and (2) protein regulations involved in tumors' cellular function and biological processes (tumour cell adhesion, apoptosis, glycogenesis and glycolysis, metastasis, angiogenesis, and protein synthesis and energy metabolism) in cancer cells. However, most of these results were derived from in vitro studies which are insufficient and less convincing since all these experiments were performed in an artificial environment. Thus, pre-clinical study using experimental cancer animal model is needed to determine the pharmacological, toxicological as well as anti-tumor effects of Phyllanthus, to provide more information on the safety usage and effectiveness of this plant against cancer.

\section{Conclusion}

In a conclusion, the Phyllanthus plant possesses multiple medicinal properties against viruses and cancer cells. Further investigations into the antiviral and anticancer properties of Phyllanthus are required to provide more information on the safe use and effectiveness of this plant. This may create opportunities for the plant to, not only be designed and developed as antiviral and anticancer agents, but also as a dietary supplement for the prevention of disease.

\section{References}

1. Cragg GM, Newman DJ. Plants as a source of anticancer agents. J Ethnopharmacol 2005; 100:72-79.

2. Ji HF, Li XJ, Zhang HY. Natural products and drug discovery. EMBO Reports 2009; 10:194-200.

3. Vincent TL, Gatenby RA. An evolutionary model for initiation, promotion, and progression in carcinogenesis. Int J Oncol 2008; 32:729-737.

4. van Agtmael MA, Eggelte TA, van Boxtel CJ. Artemisinin drugs in the treatment of malaria: from medicinal herb to registered medication. Trends Pharmacol Sci 1999; 20:199.

5. Butler MS. The Role of Natural Product Chemistry in Drug Discovery. J. Nat. Prod 2004; 67(12):2141-2153.

6. Lee $C D, O t t M$, Thyagarajan $S P$, et al. Phyllanthus amarus down-regulates hepatitis $B$ virus mRNA transcription and replication. Eur J Clin Invest 1996; 26:1069-1076. 
7. Burkill, IH. A dictionary of the economic products of Malay Peninsula. Art Printing Works, Kuala Lumpur 1996; pp 1748-1749.

8. Tang YQ, Jaganath I, Manikam R, et al. Phyllanthus Suppresses Prostate Cancer Cell, PC-3, Proliferation and Induces Apoptosis through Multiple Signalling Pathways (MAPKs, PI3K/Akt, NF B, and Hypoxia). Evid Based Complement Alternat Med 2013; e609581.

9. Lee $\mathrm{SH}$, Jaganath IB, Wang SM, et al. Antimetastatic effects of Phyllanthus on human lung (A549) and breast (MCF-7) cancer cell lines. PloS One 2011; 6:e20994.

10. Ramadasan K and Harikumar KB. Phyllanthus Species: Scientific Evaluation and Medicinal Applications (Traditional Herbal Medicines for Modern Times). 1st Ed. CRC Press; 2011.

11. Dhongade $\mathrm{H}$ and Chandewar AV. A review on pharmacognostical, Phytochemical, Pharmacological properties of Phyllanthus amarus. IJBAR. 2013; 4(5):280-288.

12. Calixto JB, Santos ARS, Yunes RA. A review of the plants of the genus Phyllanthus: their chemistry, pharmacology, and therapeutic potential. Med Res Rev 1998; 18: 225-258.

13. Taylor L. Technical Data Report for Chancap Piedra Stone Breaker (Phyllanthus niruri). In:Herbal: 2003.

14. Etta H. Effects of Phyllanthus amarus on litter traits in albino rats. Sci Res Essay 2008; 3: 370-372.

15. Mazumder A, Mahato A, Mazumder R. Antimicrobial potentiality of Phyllanthus amarus against drug resistant pathogens. Nat Prod Res 2006; 20:323-326.

16. Ott M, Thyagarajan S, Gupta S. Phyllanthus amarus suppresses hepatitis $B$ virus by interrupting interactions between HBV enhancer I and cellular transcription factors. Eur J Clin Invest 1997; 27:908-915.

17. Lee SH, Tang YQ, Rathkrishnan A, et al. Effects of cocktail of four local Malaysian medicinal plants (Phyllanthus spp.) against dengue virus 2. BMC Comp Alt Med 2013; 13:192.

18. Lee CD, Ott $M$, Thyagarajan $S$, et al. Phyllanthus amarus down-regulates hepatitis $B$ virus mRNA transcription and replication. Eur J Clin Invest 2003; 26:1069-1076.

19. Álvarez ÁL, del Barrio G, Kourí V, et al. In vitro antiherpetic activity of an aqueous extract from the plant Phyllanthus orbicularis. Phytomedicine 2009; 16:960-966.

20. Yang CM, Cheng HY, Lin TC, et al. Acetone, ethanol and methanol extracts of Phyllanthus urinaria inhibit HSV-2 infection in vitro. Antiviral res 2005; 67:24-30.

21. Cheng HY, Yang CM, Lin TC, et al. Excoecarianin, Isolated from Phyllanthus urinaria Linnea, Inhibits Herpes Simplex Virus Type 2 Infection through Inactivation of Viral Particles. Evid Based Comp Alt Med 2011; e259103.

22. Fernandez Romero J, Del Barrio Alonso G, Romeu Alvarez B, et al. In vitro antiviral activity of Phyllanthus orbicularis extracts against herpes simplex virus type 1. Phytother. Res. 2003; 17:(980-982); 980-982.

23. Álvarez A, Diñeiro $Y$, del Barrio $G$, et al. Bioactivityguided separation of anti HSV-2 and antioxidant metabolites from the plant Phyllanthus orbicularis. Planta Med 2009; 75:(PF9); PF9.

24. Qu C, Lai ZC, Pei Y, et al. Study on the Anti-HSV Activity of Crude Extract from Phyllanthus emblica in vitro. Lishizhen Medicine and Materia Medica Research 2010; 4:(007); 007.

25. Yang CM, Cheng HY, Lin TC, et al. Hippomanin a from acetone extract of Phyllanthus urinaria inhibited HSV-2 but not HSV-1 infection in vitro. Phytother. Res. 2007; 21:1182-1186.

26. Xiang $Y$, Pei $Y, Q u$ C, et al. In vitro Anti-Herpes Simplex Virus Activity of 1, 2, 4, 6-Tetra-O-galloyl- $\beta$-d-glucose from Phyllanthus emblica L.(Euphorbiaceae). Phytother. Res. 2011; 25:975-982.

27. Yang CM, Cheng HY, Lin TC, et al. The in vitro activity of geraniin and 1, 3, 4, 6-tetra- O-galloyl- $\beta$ - $d$ glucose isolated from Phyllanthus urinaria against herpes simplex virus type 1 and type 2 infection. $J$ ethnopharmacol 2007; 110:555-558.

28. Tan WC, Jaganath IB, Manikam R and Sekaran SD. Evaluation of four local Malaysian Phyllanthus species against herpes simplex viruses and possible antiviral targets. Int J Med Sci 2013; 10(13):1817-1829.

29. Pei Y, Xiang YF, Chen JN, et al. Pentagalloylglucose downregulates cofilin1 and inhibits HSV-1 infection. Antiviral res 2011; 89:98-108.

30. Álvarez ÁL, Dalton KP, Nicieza I, et al. Bioactivityguided Fractionation of Phyllanthus orbicularis and Identification of the Principal Anti HSV-2 Compounds. Phytother. Res 2012; 26:1513-1520.

31. Whitehorn J, Simmons CP. The pathogenesis of dengue. Vaccine 2011; 29:7221-7228.

32. Wan SW, Lin CF, Yeh TM, et al. Autoimmunity in dengue pathogenesis. J Formos Med Assoc 2012; 112:3-11.

33. Schmitz J, Roehrig J, Barrett A, et al. Next generation dengue vaccines: a review of candidates in preclinical development. Vaccine 2011; 29:7276-7284.

34. Herrero LJ, Zakhary A, Gahan ME, et al. Dengue virus therapeutic intervention strategies based on viral, vector and host factors involved in disease pathogenesis. Pharmacol Ther 2012; 137:266-282.

35. Klawikkan $\mathrm{N}$, Nukoolkarn $\mathrm{V}$, Jirakanjanakir $\mathrm{N}$, et al. Effect of Thai medicinal plant extracts against Dengue virus in vitro. MU J Pharm 2011; 38:13-18.

36. Tan W, Lee $S$, Tang $Y$, et al. Antiviral effects of a Malaysia medicinal plant (Phyllanthus). JUMMEC 2013:(25); 25.

37. Jeyasankar A, Elumalai K. Larvicidal activity of Phyllanthus emblica Linn.(Euphorbiaceae) leaf extracts against important human vector mosquitoes (Diptera: Culicidae). Asian Pac J Trop Dis 2012; 2:S399-S403.

38. Shoeb M. Anticancer agents from medicinal plants. Bangladesh J Pharmacol 2006; 1:35-41.

39. Holt GA, Chandra A. Herbs in the modern healthcare environment-An overview of uses, legalities, and the role of the healthcare professional. Clin Res Reg Aff 2002; 19:83-107.

40. Tang YQ, Jaganath IB, Sekaran SD. Phyllanthus spp. induces selective growth inhibition of PC-3 and 
MeWo human cancer cells through modulation of cell cycle and induction of apoptosis. PLoS One 2010; 5:e12644.

41. Huang ST, Yang RC, Lee PN, et al. Anti-tumor and anti-angiogenic effects of Phyllanthus urinaria in mice bearing Lewis lung carcinoma. Int Immunopharmacol 2006; 6:870-879.

42. Huang ST, Yang RC, Pang JHS. Aqueous extract of Phyllanthus urinaria induces apoptosis in human cancer cells. Am J Chin Med 2004; 32:(175-183); 175-183.

43. Sureban SM, Subramania D, Rajendran P, et al. Therapeutic effects of Phyllanthus species: induction of TNF- $\alpha$-mediated apoptosis in HepG2 hepatocellular carcinoma cells. Am J Pharmacol Toxicol 2007; 1:65.

44. Zhong $\mathrm{Z}$, Huang J, Liang $\mathrm{H}$, et al. The effect of gallic acid extracted from leaves of Phyllanthus emblica on apoptosis of human hepatocellular carcinoma BEL7404 cells. Zhong yao cai 2009; 32:(1097); 1097.

45. Hsieh T, Wu JM. Differential effects on growth, cell cycle arrest, and induction of apoptosis by resveratrol in human prostate cancer cell lines. Exp Cell Res 1999; 249:109-115.

46. Evan GI, Vousden KH. Proliferation, cell cycle and apoptosis in cancer. Nature 2001; 411:342-348.

47. Collins NL, Reginato MJ, Paulus JK, et al. G1/S cell cycle arrest provides anoikis resistance through Erk-mediated Bim suppression. Mol Cell Biol 2005; 25:5282-5291.

48. Massagué J. G1 cell-cycle control and cancer. Nature 2004; 432:298-306.

49. Dolcet $X$, Llobet $D$, Pallares J, et al. NF-kB in development and progression of human cancer. Virchows Archiv 2005; 446:475-482.

50. Zhang HJ, Zhao W, Venkataraman S, et al. Activation of matrix metalloproteinase-2 by overexpression of manganese superoxide dismutase in human breast cancer MCF-7 cells involves reactive oxygen species. J Biol Chem 2002; 277:20919-20926.

51. Tseng $\mathrm{HH}$, Chen PN, Kuo WH, et al. Antimetastatic Potentials of Phyllanthus urinaria L on A549 and Lewis Lung Carcinoma Cells via Repression of MatrixDegrading Proteases. Integr Cancer Ther 2012; 11:267-278.

52. Xia SH, Wang J, Kang JX. Decreased n-6/n-3 fatty acid ratio reduces the invasive potential of human lung cancer cells by downregulation of cell adhesion/ invasion-related genes. Carcinogenesis 2005; 26:779784.

53. Huang ST, Wang CY, Yang RC, et al. Ellagic acid, the active compound of Phyllanthus urinaria, exerts in vivo anti-angiogenic effect and inhibits MMP-2 activity. Evid Based Comp Alt Med 2011:e215035.

54. Ho HH, Chang CS, Ho WC, et al. Anti-metastasis effects of gallic acid on gastric cancer cells involves inhibition of NF-KB activity and downregulation of PI3K/AKT/small GTPase signals. Food Chem Toxicol 2010; 48:2508-2516.

55. Ngamkitidechakul C, Jaijoy K, Hansakul P, et al. Antitumour effects of Phyllanthus emblica L.: induction of cancer cell apoptosis and inhibition of in vivo tumour promotion and in vitro invasion of human cancer cells. Phytother. Res. 2010; 24:14051413.

56. Tseng $\mathrm{HH}$, Chen PN, Kuo WH, et al. Antimetastatic Potentials of Phyllanthus urinaria L on A549 and Lewis Lung Carcinoma Cells via Repression of MatrixDegrading Proteases. Integr Cancer Ther 2012; 11:267-278.

57. Lu KH, Yang HW, Su CW, et al. Phyllanthus urinaria suppresses human osteosarcoma cell invasion and migration by transcriptionally inhibiting u-PA via ERK and Akt signaling pathways. Food Chem toxicol 2012; 52:193-199. 